\title{
Call blocking in multi-services systems on one transmission link.
}

\author{
Myriam DELAIRE and Gérard HEBUTERNE \\ Institut National des Télécommunications, Dept. RST \\ Contact: Gerard.Hebuterne@int-evry.fr
}

\begin{abstract}
Future broadband integrated services digital networks (B-ISDN) are expected to use the Asynchronous Transfer Mode (ATM) technology and support multiple services. In the multiservices context, three Connection Admission Control (CAC) strategies with guaranteed Grade of Services (GoS)are presented: Complete Sharing (CS), Complete Sharing with Equalization (CSE) and Routes Separation (RS). The methods are described and compared in order to identify their most suitable operating regions. A mapping scheme selecting the appropriate CAC method according to traffic conditions and environments is then deduced. Performance results are reported for a set of reference scenarios.
\end{abstract}

Key words: ATM, multiple services, CAC, equalization, call blocking

\section{Introduction.}

The presence of different emerging services with specific GOS in future ATM networks requires the development of new services acceptance models. There are three levels of acceptance: cell level, burst level and call level (see [ROB92, RMV96]). Most studies have focussed on cell and burst levels. Studies on call level and multi-rate traffic are not as common. The thrust of this work is to conduct the analysis at the call level in the presence of multi rate traffic sources.

The connection oriented property of ATM suggests the allocation of part of the resource for the entire connection life. Assuming that an equivalent bandwidth characterization of Variable Bit Rate (VBR) sources is adopted, only Constant Bit Rate (CBR) calls are considered. Refer to [RTG94] for the derivation of the equivalent bandwidth for VBR traffic. Only one unique transmission link receiving input traffic resulting from the superposition of $N$ traffic classes is considered in this study. Three CAC strategies are defined for this multi service system where each class requires a different GoS. Exact results are provided for two of these methods while an approximate solution is reported for the remaining scheme.

The original version of this chapter was revised: The copyright line was incorrect. This has been corrected. The Erratum to this chapter is available at DOI: 10.1007/978-0-387-35353-1_28

D. Kouvatsos (ed.), Performance Analysis of ATM Networks

(C) IFIP International Federation for Information Processing 2000 
Much work has been devoted to the issue of determining GoS allocation to multiservices connections [SkR93, Ros95], etc. Here, we extend this approach by showing how to give the same GoS to connections with different bit rate requirement.

\section{Description of call admission methods.}

Arriving calls are accepted only if the available link capacity is greater than or equal to the required call bit rate. A call of class $i(i=1, \ldots, N)$ with bit rate requirement $d_{i}$ is blocked with a probability $B_{i}$. Further, the total capacity is denoted as $C$ while $C_{R}$ represents the available resource capacity. Given these notations, the three methods developed in [RKK88] are described.

\subsection{Complete Sharing (CS) method.}

In this most often used method, the transmission link can be assigned to any call type or class, see Figure 1.

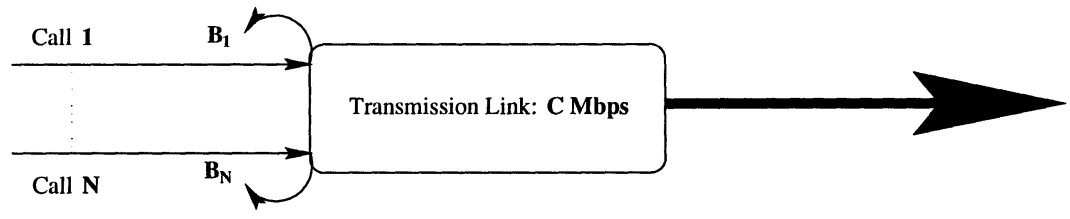

Figure 1: Complete Sharing

The condition for call acceptance is as follows:

Call Admission Control (CAC) 1 (CS)

An arriving call of class $i$ will be accepted if and only if the available link capacity $C_{R}$ is greater than or equal to the bit rate requirement $d_{i}$.

$$
C_{R} \geq d_{i}
$$

\subsection{Complete Sharing with Equalization (CSE) method.}

The CS method exhibits the drawback of causing higher blocking rates to calls with higher bit rate requirements $d_{i}$ while favoring calls with lower bit rate $d_{j}\left(B_{i}>B_{j}\right)$. In order to provide fair access to all classes, despite their different bit rate requirements, an equalization mechanism can be introduced.

\subsubsection{Definition of equalization mechanism.}

The rule for equalizing call blocking probabilities presented in [RTG94] is repeated below: 


\section{MeChaNism 1 (Equalization)}

Given $N$ different classes, call blocking probabilities $B_{i}$ are equal if and only if an acceptance threshold $\theta$ set to $\max d_{i}$ is used. (recall that $d_{i}$ is the bit rate requirement of class $i$ )

$$
\theta=\max _{i}\left\{d_{i}\right\}
$$

Note that the threshold $\theta$ is the same for all classes. An algorithm for call acceptance, based on the complete sharing strategy with the equalization mechanism is defined in the next section.

\subsubsection{CS with Equalization of call blockings = Equalization.}

With the equalization mechanism only calls from the class with higher bit rate requirement can access the whole link capacity see Figure 2.

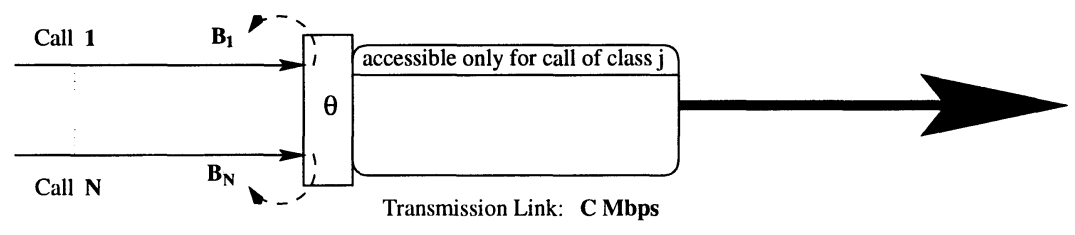

Figure 2: Complete Sharing with Equalization

The call acceptance criterion becomes as follows:

Call Admission Control (CAC) 2 (CSE)

An arriving call of class $i$ will be accepted, if and only if the available link capacity $C_{R}$ is greater than or equal to the threshold $\theta$.

$$
C_{R} \geq \theta
$$

\subsection{Route Separation (RS) method.}

In this strategy, the link is divided into resource sub-groups. There are as many sub-groups as call classes, with $C_{i}$ as the link capacity of class $i$ sub-group, see Figure 3.

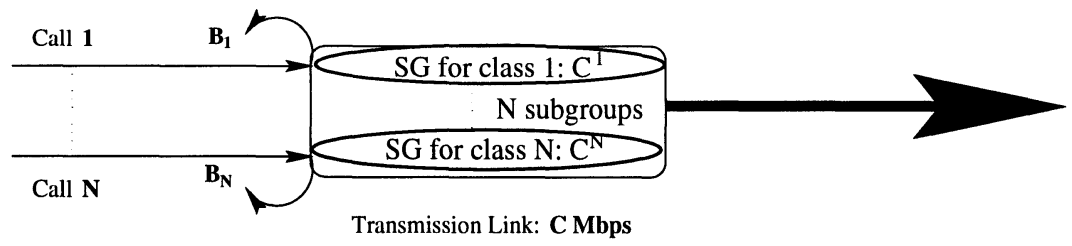

Figure 3: Routes Separation

Thus, the condition of acceptance is given as: 
Call Admission Control (CAC) 3 (RS)

An arriving call of class $i$ will be accepted if and only if the available capacity $C_{R}^{i}$ of the class $i$ sub-group is greater or equal to the bitrate requirement $d_{i}$.

$$
C_{R}^{i} \geq d_{i} \text { with } \sum_{i} C_{R}^{i} \leq C
$$

This method is also refered to as Class Limitation, Complete Separation, Complete Partitioning, and so on in the litterature.

\section{Comparison of access control strategies.}

In call level CAC, the key GoS parameters are: bitrate requirement, arrival rate, holding time and blocking probability. Type $i$ arrival traffic is assumed to follow a Poisson process with rate $\lambda_{i}$. During the holding time of a class $i$ call, assumed to have a negative-exponential distribution function with mean $1 / \mu_{i}$, a constant bit rate $d_{i}$ is reserved for this call until completetion.

A scenario with a mix of two different traffic classes on the same link is analyzed. The total offered traffic is kept constant. Link capacity is increased to achieve a blocking probability $B_{i}$ less than or equal to $1 \%$ while keeping the product $\rho . C$ (that is the total offered traffic) as constant. Variable $\rho$ represents the utilization factor, which must be decreased accordingly. This guarantees operation under the $1 \%$ blocking condition. Bit rates for each class as well as the offered traffic ratio are known. The normalized offered traffic from a class $i$ call is denoted as $A_{i}$. The ratio $A_{1} / A_{2}$ varies from 0.01 to 100 .

Since we operate in regions of low blocking ratios (less than 1\%), no distinction is made between offered and carried traffics.

$$
\rho \cdot C=\sum_{i} A_{i} \text { with } A_{i}=\frac{\lambda_{i} \cdot d_{i}}{\mu_{i}}
$$

In this section, RATIO represents $\frac{A_{1}}{A_{2}}$, where $A_{i}$ is the offered traffic for a call of class $i$. Parameters are given in the following Table 1:

\begin{tabular}{|c|c|c|}
\hline Calls & $d_{i}$ & $A_{i}$ \\
\hline \hline Class 1 & $d_{1} \mathrm{~Kb} / \mathrm{s}$ & $\frac{O \text { fferedtraffic }}{1+1 / R A T I O}$ \\
\hline Class 2 & $d_{2} \mathrm{~Kb} / \mathrm{s}$ & $\frac{\text { Offeredtraffic }}{1+R A T I O}$ \\
\hline
\end{tabular}

Table 1: Parameters of the comparison

The bandwidth values are discretized: a basic bandwidth unit $\Delta C$ is defined using the $g c d^{1}$ function:

$$
\Delta C=\operatorname{gcd}\left\{d_{i}\right\} \quad 1 \leq i \leq N
$$

\footnotetext{
${ }^{1}$ gcd means greatest common divisor.
} 
The maximum number of available basic bandwidth units is denoted as $M$ and the number of required basic bandwidth units (by class $i$ ) is $\delta_{i}$. For the recursive solution, states of the traffic model are defined by $m(m=0,1, \ldots, M)$, bandwidth units.

$$
M \equiv \frac{C}{\Delta C} \quad \delta_{i} \equiv \frac{d_{i}}{\Delta C}
$$

\subsection{Analysis of the Complete Sharing method}

Two approaches may be used in order to obtain exact call blocking probabilities for this access control strategy, namely :

- The first one is based on a product form solution as described in [EMi73]. The system state is defined as the number of accepted calls from each class $\left(n_{1}, \ldots, n_{N}\right)$. The multi-dimensional state space has as many dimensions as the number of traffic classes. This leads to the typical state explosion problem. An example of the state space is depicted in Figure 4.

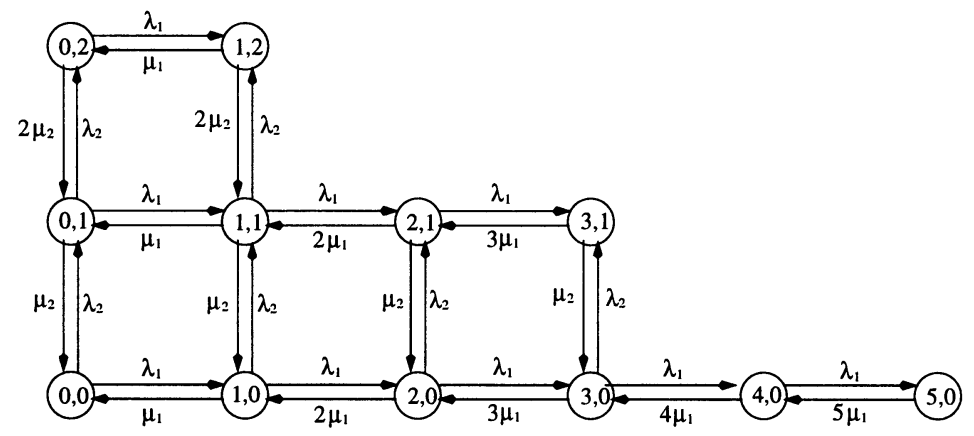

Figure 4: State space example for the product form solution $\left(N=2, C_{1}=C / 5\right.$, $\left.C_{2}=C / 2.5\right)$

- In the second approach, the multi-dimensional state space is mapped into a one-dimensional state space without affecting the resulting blocking probabilities. These results are given using a recursive solution according to the algorithm proposed in [DRo87]. This method, suitable for alleviating the state explosion problem, will be explained in the following paragraph. A state diagram is given in Figure $\mathbf{5}$.

The unnormalized state probabilities can be derived using the following recursive algorithm:

$$
\tilde{p}(m)=\begin{array}{ll}
1 & m=0 \\
0 & m<0 \\
\frac{1}{m} \sum_{i=1}^{N} \tilde{p}\left(m-\delta_{i}\right) \cdot \delta_{i} \cdot \frac{\lambda_{i}}{\mu_{i}} & 0<m \leq M
\end{array}
$$




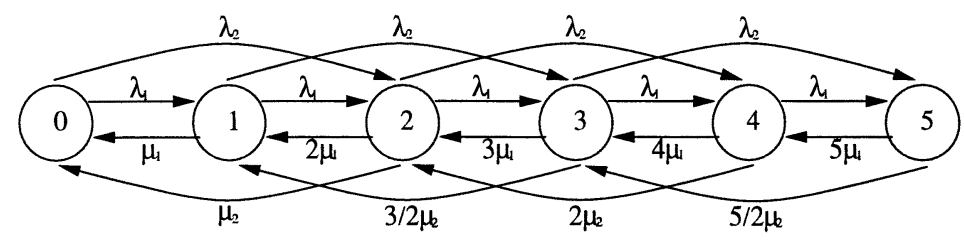

Figure 5: State space reduction for recursive solution $\left(M=5, \delta_{1}=1, \delta_{2}=2\right)$

After normalization, state probabilities and blocking probability for calls of class $i$ are obtain as:

$$
p(m)=\tilde{p}(m) \cdot\left(\sum_{m=0}^{M} \tilde{p}(m)\right)^{-1} \quad B_{i}=\sum_{m=M-\delta_{i}+1}^{M} p(m)
$$

Using Equation 8 combined with Equations 4 through 7, we can derive the total capacity of the link under the constraint of $1 \%$ call block and thus the total offered traffic for the link.

$$
\max _{i}\left(b_{i}\right)=\max _{i}\left(\sum_{m=M-\delta_{i}+1}^{M} p(m)\right) \leq 0.01
$$

The total capacity of the link can be obtained by $C=M \cdot \Delta C$ as the following equation:

$$
\rho M=\sum_{i} \rho_{i} \delta_{i} \quad \text { or } \quad \rho \frac{C}{\Delta C}=\sum_{i} \rho_{i} \frac{d_{i}}{\Delta C}
$$

\subsection{Analysis of the Equalization Method}

Since exact solutions for the CSE method do not exist, an approximation, based on the recursive solution, is proposed in [ROB92]. The states are defined as before by the number of occupied basic bandwidth units, but the state space description is slightly different since some of the transitions between states disappear following the introduction of the threshold $\theta$. Figure 6 gives the state space diagram for the Equalization method.

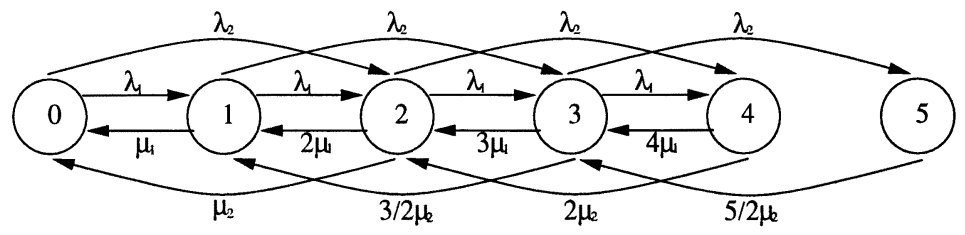

Figure 6: State space example for recursive solution with equalization $(M=5$, $\delta_{1}=1, \delta_{2}=2$ ) 
Analysis of the equalization mechanism requires the introduction of a new function $G_{i}\left(m-\delta_{i}\right)$ integrating the notion of threshold in call acceptance management. It is defined as:

$$
G_{i}\left(m-\delta_{i}\right)=\begin{array}{ll}
\delta_{i} & m \cdot \Delta C \leq C-\theta \\
0 & m \cdot \Delta C>C-\theta
\end{array}
$$

The unnormalized state probabilities can be obtained using the following recursion algorithm Equation 7), leading to an approximate solution:

$$
\tilde{p}(m)=\begin{array}{ll}
1 & m=0 \\
0 & m<0 \\
\frac{1}{m} \sum_{i=1}^{N} \tilde{p}^{*}\left(m-\delta_{i}\right) \cdot G_{i}\left(m-\delta_{i}\right) \cdot \frac{\lambda_{i}}{\mu_{i}} & 0<m \leq M
\end{array}
$$

After normalization, state probabilities $p^{*}(m)$ and blocking probability $B_{i}^{*}$ for class $i$ calls are:

$$
B_{i}^{*}=\sum_{m=\min \left\{M-\delta_{i},\left(C-\theta_{i}\right) / \Delta C\right\}+1}^{M} p^{*}(m) \quad \text { with } \quad p^{*}=\tilde{p^{*}}(m) \cdot \sum_{m=0}^{M} \tilde{p^{*}}(m)
$$

Total link capacity and offered bit rate can be evaluated using the same steps as in the CS method.

\subsection{Route separation.}

In this strategy, each call class is assigned to its dedicated transmission link sub-group. To obtain the total link capacity, link capacity from all sub-groups must be summed up. Since mixing or multiplexing of classes does not occur in RS, call blocking probability for each class can be obtained directly from the Erlang loss formula. Thus call blocking as a function of number of calls of class $i, N_{i}$, is easily obtained as follow:

$$
B_{i}=E\left(\rho_{i}, N_{i}\right)=\frac{\rho_{i}^{N_{I}} / N_{i} !}{\sum_{j \leq N_{i}} \rho_{i}^{j} / j !} \quad \text { with } \quad \rho_{i}=\frac{\lambda_{i}}{\mu_{i}}
$$

Total link capacity is calculated directly through:

$$
C=\sum_{i} N_{i} \cdot d_{i}=\sum_{i} C_{i}
$$

\section{Numerical comparison of the control algorithms}

The selected bandwidth unit is $100 \mathrm{Kbps}$. Experiments are conducted with offered traffic of $25 \mathrm{Mbps}$ and $155 \mathrm{Mbps}$, typical ATM transmission link capacities. 
In this section numerical results are displayed corresponding to the previous comparison. Curves show carried traffic when ensuring $B_{1}$ and $B_{2}$ less than or equal to $1 \%$.

\subsection{Mbps links.}

Four tests with different bit rate requirements were conducted for each control strategy for a given offered traffic load. The initial objective was to extract from these tests the most appropriate control strategy for each scenario. Total link traffic is fixed at $25 \mathrm{Mbps}$, and test parameters are given in Table 2.

\begin{tabular}{|c|c|c|c|}
\hline Calls & TEST 1 & TEST 2 & TEST 3 \\
\hline \hline Class 1 & $10 \mathrm{~Kb} / \mathrm{s} \Rightarrow 0.1$ unit & $200 \mathrm{~Kb} / \mathrm{s} \Rightarrow 2$ units & $1 \mathrm{Mb} / \mathrm{s} \Rightarrow 10$ units \\
\hline Class 2 & $50 \mathrm{~Kb} / \mathrm{s} \Rightarrow 0.5$ unit & $2 \mathrm{Mb} / \mathrm{s} \Rightarrow 20$ units & $3 \mathrm{Mb} / \mathrm{s} \Rightarrow 30$ units \\
\hline
\end{tabular}

Table 2: Tests with $25 \mathrm{Mb} / \mathrm{s}$

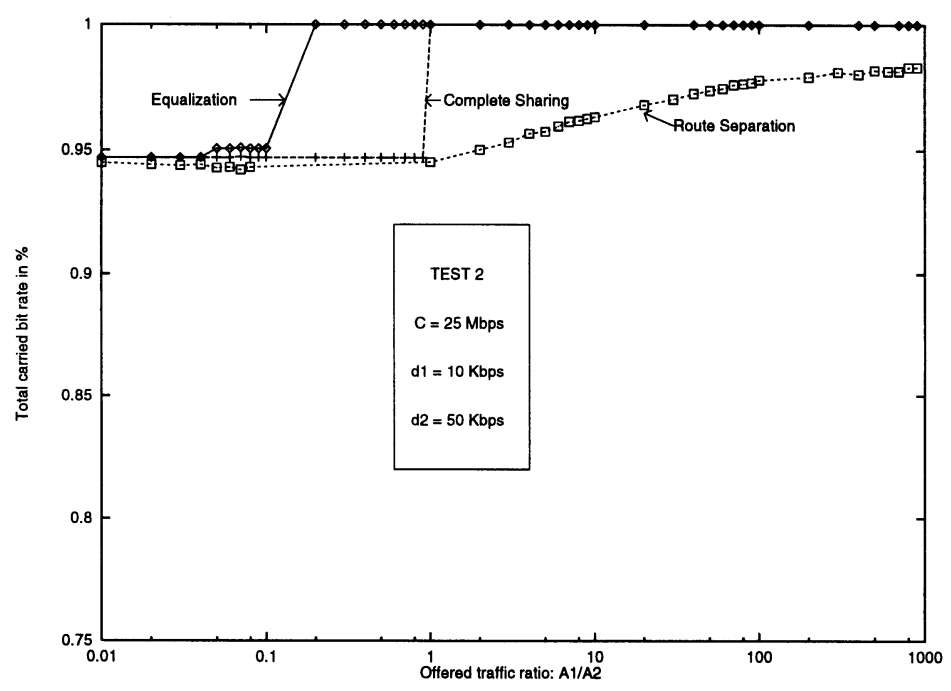

Figure 7: First test with $25 \mathrm{Mb} / \mathrm{s}$

Results from these tests are reported in Figures $\mathbf{7}$ through $\mathbf{8}$ and following observations can be made.

- Complete sharing with equalization gives always better results than the two others methods.

- Simple complete sharing gives the same results as Equalization when traffic from the class with greater bit rate requirement is ten times greater than 
that of the other traffic class. Over all test cases, Equalization is always better, for higher ratios.

- Routes separation provides in general worse results than complete sharing. It is only interesting for the first test and when $A_{1}$ is much greater (a factor of one hundred or more) than $A_{2}$.

- For all bit rate requirements the results remain consistent with Equalization achieving best performance experiments across all test.
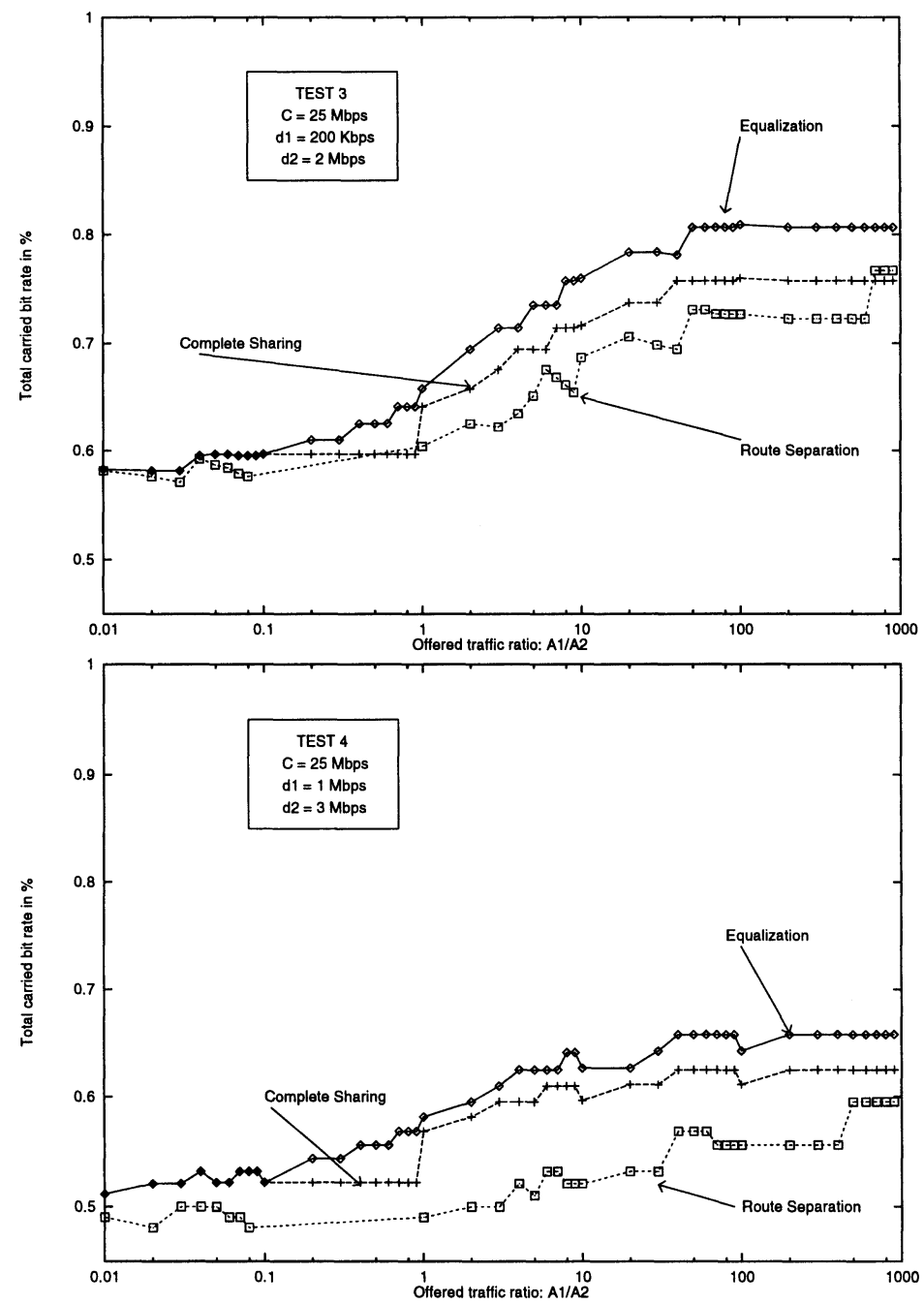

Figure 8: Second and Third tests with $25 \mathrm{Mb} / \mathrm{s}$ 


\subsection{Mbps links}

Total link traffic is fixed at $155 \mathrm{Mbps}$ for these three experiments. Other parameters are described in Table 3.

\begin{tabular}{|c|c|c|c|}
\hline Calls & TEST 1 & TEST 2 & TEST 3 \\
\hline \hline Class 1 & $100 \mathrm{~Kb} / \mathrm{s} \Rightarrow 1$ unit & $200 \mathrm{~Kb} / \mathrm{s} \Rightarrow 2$ units & $1 \mathrm{Mb} / \mathrm{s} \Rightarrow 10$ units \\
Class 2 & $500 \mathrm{~Kb} / \mathrm{s} \Rightarrow 5$ units & $2 \mathrm{Mb} / \mathrm{s} \Rightarrow 20$ units & $3 \mathrm{Mb} / \mathrm{s} \Rightarrow 30$ units \\
\hline
\end{tabular}

Table 3: Tests with $155 \mathrm{Mb} / \mathrm{s}$

The results are displayed in Figures 9 through 10. Concerning the tests at $155 \mathrm{Mbps}$, the same conclusions can be drawn. Complete sharing with call blocking equalization performs better than the two other methods, while routes separation provides the worst results.

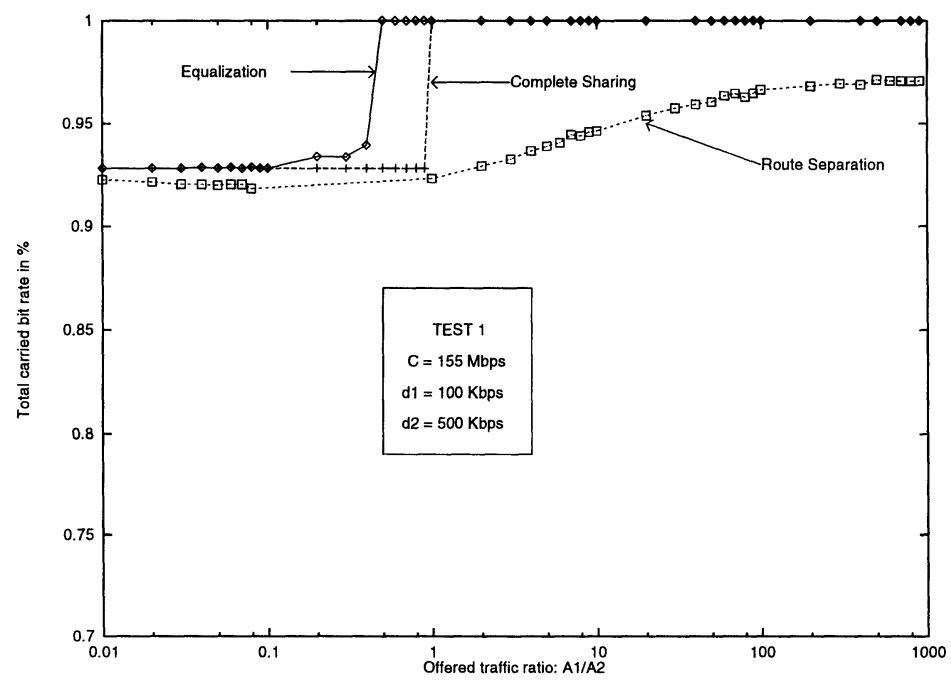

Figure 9: First test with $155 \mathrm{Mb} / \mathrm{s}$.

\section{Accuracy of the Equalization method.}

As seen in section 3, exact call blocking probabilities can be obtained using a recursive solution in the case of the Complete Sharing mechanism. But no exact solutions exist for Complete sharing method with equalization. Several approximations were proposed in [RMV96]. The most accurate (which is used here) is based on the recursive solution with the introduction of the threshold notion. Tests were conducted in order to control the accuracy of the approximation. A simulation is used to assess the accuracy of the approximation. 
- First, the call blocking probabilities are observed for varying holding time ratios.

- In a second step, one intends to observe the influence of the relative loads.

- The last test attempts at justifying the interest of the equalization method.
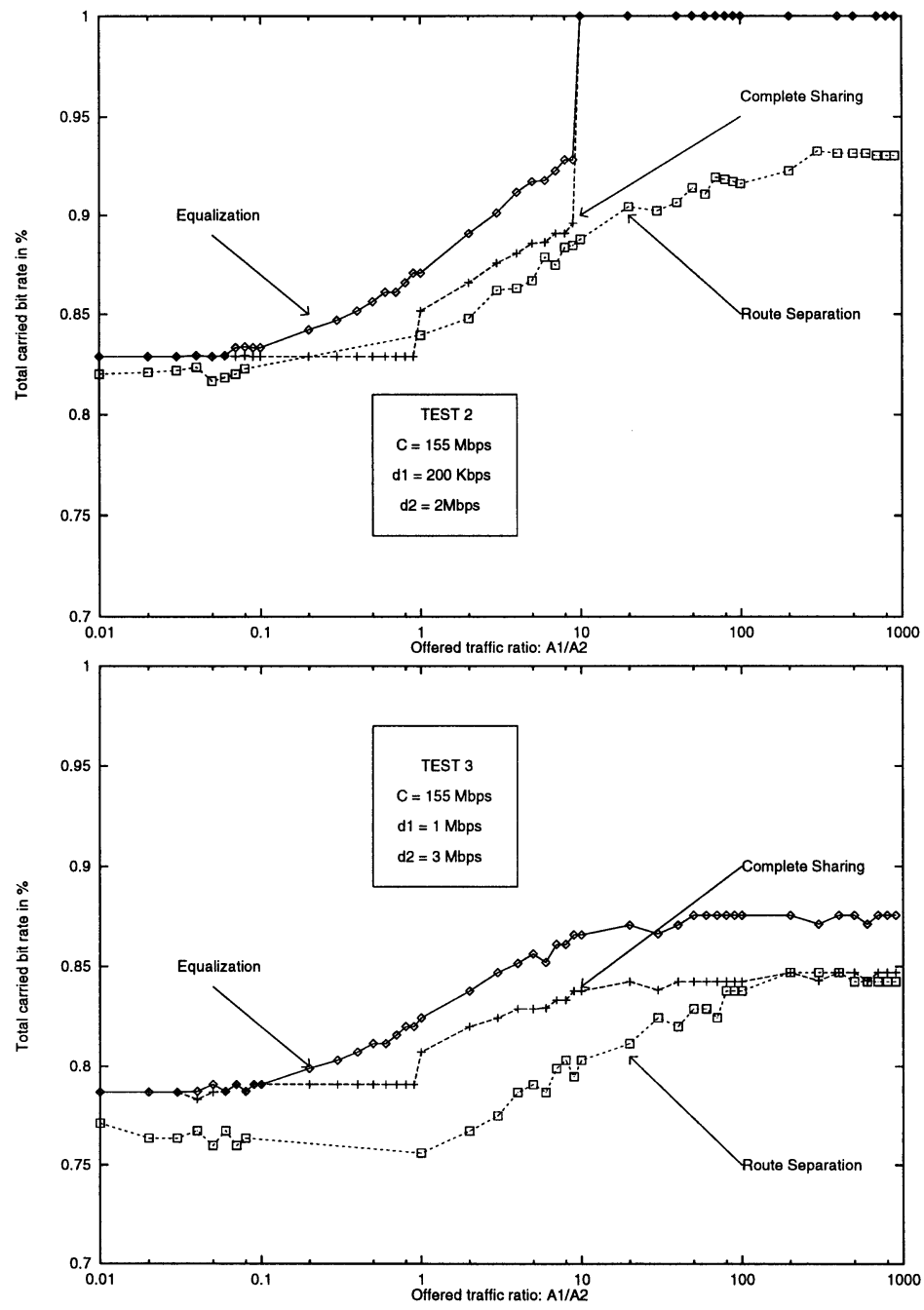

Figure 10: Second and Third tests with $155 \mathrm{Mb} / \mathrm{s}$.

All tests were conducted on one single link of constant capacity $C$ and two call classes. All parameters are given in units of bandwidth. 


\subsection{Variation of the Holding time ratio.}

In this part, the ratio of service rates varies from 0.1 to 100 . The ratio is expressed by $\frac{\mu_{0}}{\mu_{1}}$. The total link capacity is fixed: $C=30$ units.

On each experiment, we compare the blocking probabilities of each class in function of the holding time ratio - first without the Equalization mechanism (Simple Complete Sharing) and then with this fairness mechanism (Complete Sharing with Equalization).

\subsubsection{Tests description.}

Two experiments were conducted, as summarized in Table 4.

\begin{tabular}{||l|c|c||c|c|}
\cline { 2 - 5 } \multicolumn{1}{c|}{} & \multicolumn{2}{c|}{ Heavy load traffic } & \multicolumn{2}{c|}{ Low load traffic } \\
\cline { 2 - 5 } \multicolumn{1}{c|}{} & Class 0 & Class 1 & Class 0 & Class 1 \\
\hline \hline Arrival rate & $\lambda_{0}=10$ & $\lambda_{1}=5 \cdot \mu_{1}$ & $\lambda_{0}=10$ & $\lambda_{1}=\mu_{1}$ \\
\hline Services rate & $\mu_{0}=1$ & $\mu_{0} /$ ratio & $\mu_{0}=1$ & $\mu_{0} /$ ratio \\
\hline Bit rate requirement & $d_{0}=1$ & $d_{1}=5$ & $d_{0}=1$ & $d_{1}=5$ \\
\hline \hline Total traffic & \multicolumn{2}{c|}{$\rho_{H}=1.17$} & \multicolumn{2}{c|}{$\rho_{l}=0.5$} \\
\hline
\end{tabular}

Table 4: Holding time ratio variation's parameters

The two methods have been tested under the same conditions, both using the analytical approach and a simulation.

\subsubsection{Influence of the Holding times}

Results for High trafic load and Low traffic load are given in Figures 11 and 12. Simulations have been obtained by computing 5 million of events. The imprecision is lower than $10 \%$, at a $95 \%$ confidence level. The continuous lines represent results of the analytical method (the exact one for CS, the approximation for CSE), while the points are simulation results.

The curves suggest the following comments.

- On the whole, the results are in accordance. This validates the models.

- The "exact" solution for CS gives constant values for losses as the ratio varies, and so does the approximation. However, simulation results show a clear influence of the ratio $\mu_{0} / \mu_{1}$, especially for low load. The recursive solution with equalization is unable to capture this effect (which is not surprizing, since only the loads $\rho_{i}$ are input parameters for the model). The phenomenon has been already reported in [RMV96] and it has no clear explanation. 


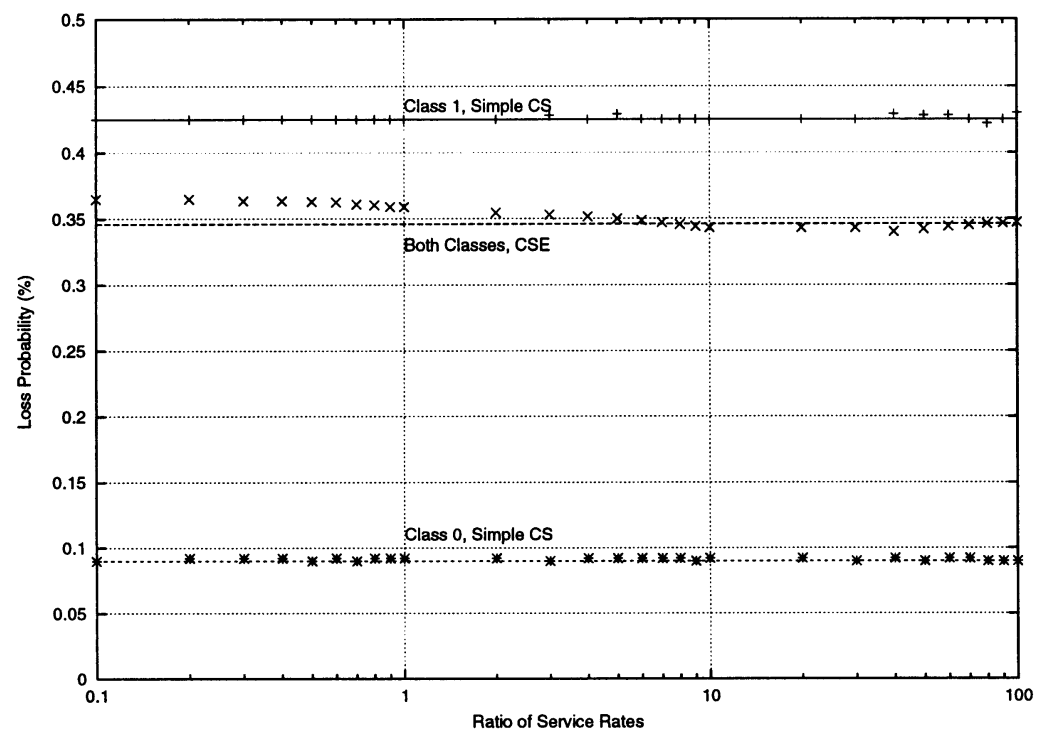

Figure 11: Approximation vs Simulation, High traffic configuration

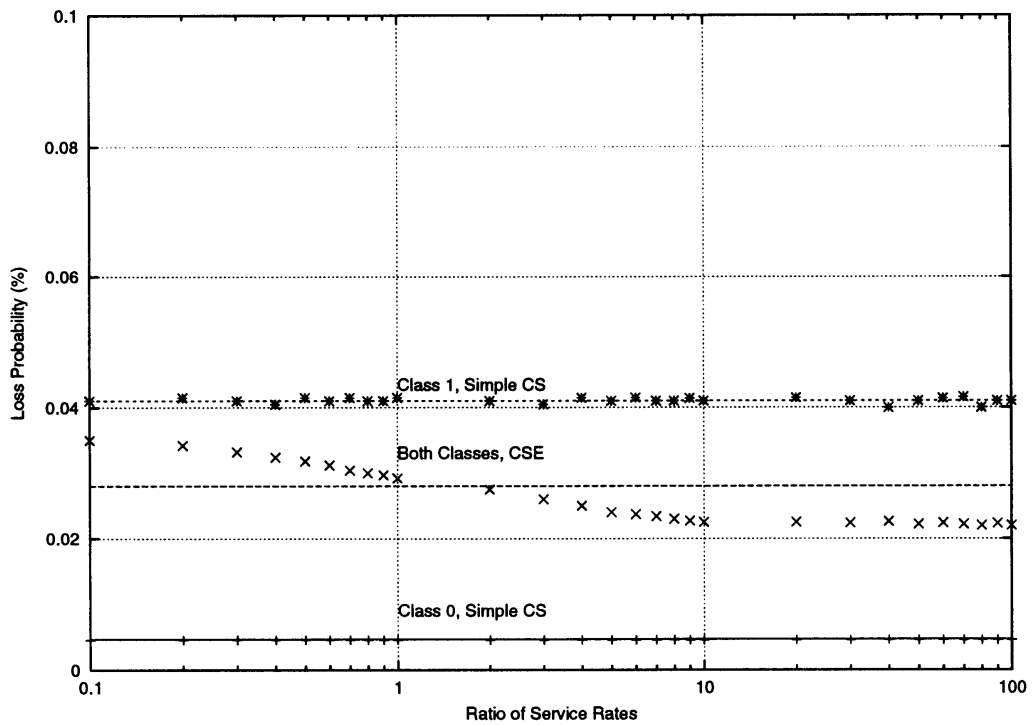

Figure 12: Approximation vs Simulation, Low traffic configuration 


\subsection{Variation of the Offered load ratio.}

In this part, the traffics $\rho_{i}$ vary in such a way that the overall load is kept constant, so as to compare their impact on the efficiency of the mechanisms. For this test, the total link capacity $C=30$ is fixed, the total carried trafic is $\rho=70 \%$. Other parameters are given in Table 6 .

\begin{tabular}{|c|c|c|}
\cline { 2 - 3 } \multicolumn{1}{c|}{} & Class 0 & Class 1 \\
\hline Service rate & $\mu_{0}=10$ & $\mu_{1}=1$ \\
\hline Bit rate requirement & $d_{0}=1$ & $d_{1}=5$ \\
\hline
\end{tabular}

Table 5: Parameters for the variation of load ratio

The analytical results are given in Figure 13. The simulation results are omitted. Anyway, they are in complete agreement with the analytical ones.

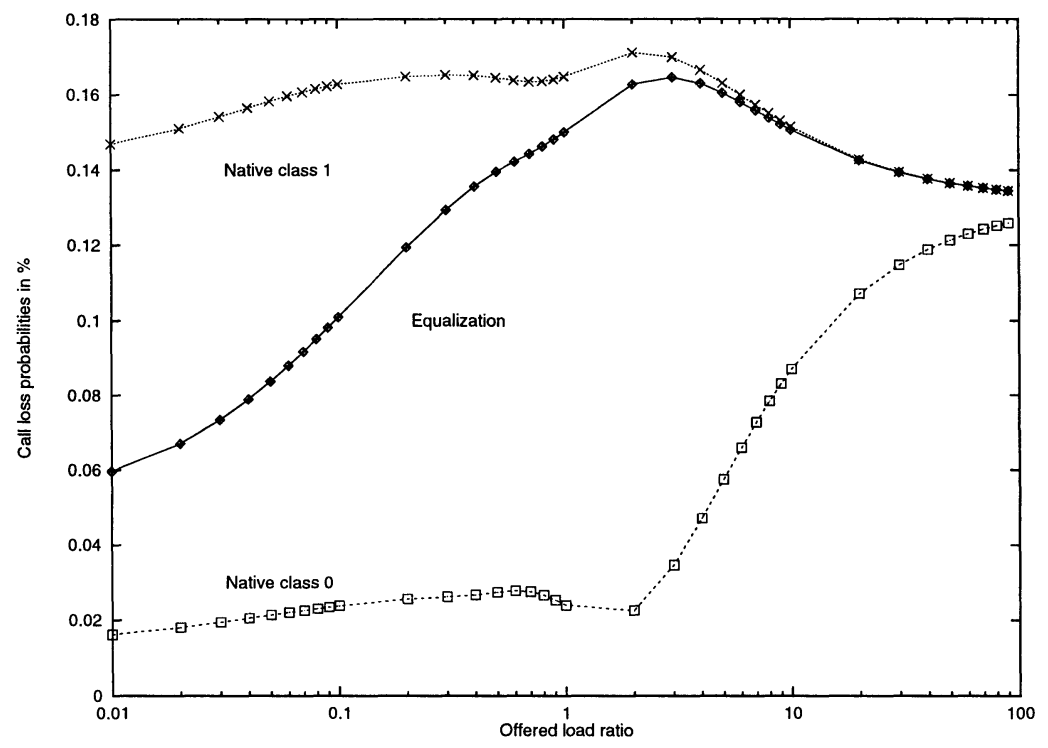

Figure 13: Influence of the ratio $\rho_{0} / \rho_{1}$. Total Load $=0.7$

\subsection{Importance of the approximation.}

To be sure that the Complete Sharing with Equalization method is necessary and gives better results than Simple Complete Sharing mechanism, we made some others tests.

- First, the offered load is increased (= total link capacity).

- Then, the bit rate required by the second class is decreased. 
- In the last test, the class 0's arrival rate is decreased.

Simulation and algorithm computing were both made. Results obtained with simulations and using model algorithms are similar with an $90 \%$ confidence interval. Thus, we only show curves using results of model results.

\subsubsection{Parameters' presentation.}

Parameters for the three tests are given in the Table 5. In this table are shown variation to provide less call losses.

We compare call losses of each class without Equalization mechanism and call losses using this fairness method.

\begin{tabular}{|c|c|c|c|c|c|c|c|c|}
\hline & \multicolumn{2}{|c|}{ Basic parameters } & \multicolumn{2}{|c|}{ 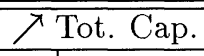 } & \multicolumn{2}{|c|}{$\searrow \lambda_{0}$} & \multicolumn{2}{|c|}{$\searrow d_{1}$} \\
\hline Class & $\overline{0}$ & 1 & 0 & 1 & 0 & 1 & 0 & $\overline{1}$ \\
\hline$\overline{\lambda_{i}=}$ & 10 & $\overline{\overline{5 \cdot \mu_{1}}}$ & $\overline{=}$ & $\overline{\bar{E}}$ & to 8 & $\overline{=}$ & $\overline{=}$ & $=$ \\
\hline$\mu_{i}=$ & 1 & $\mu_{0} /$ ratio & $=$ & $=$ & $=$ & $=$ & $\overline{=}$ & $=$ \\
\hline$d_{i}=$ & 1 & 5 & $=$ & $=$ & $=$ & $=$ & $=$ & to 4 \\
\hline Tot. Cap.: $C$ & & 30 & & $\overline{35}$ & $=$ & & & $\bar{E}$ \\
\hline
\end{tabular}

Table 6: Heavy load traffic parameters

\subsubsection{Verification results.}

Concerning results for Offered Load increase, bit rate requirement decrease and arrival rate decrease see Figures 15,16 and 17 respectively.

\section{Conclusion and further study.}

The Complete Sharing with Equalization method can achieve gains in carried bit rate of $8 \%$ compared with the complete sharing method and of $16 \%$ compared with the Routes Separation strategy. Of all the schemes, routes separations is not attractive, not only because of poor performance but also because of an increase in resource management complexity and service deployment.

Complete sharing, easier to implement, exhibits fairness problems under certain conditions as evidenced by results obtained from various tests.

Equalization and Routes Separation provide fair access to the resource. Equalization achieves better utilization of the network resource, however.

CS respects the required $\operatorname{GoS}\left(B_{1}, B_{2}\right.$ less than or equal to $\left.1 \%\right)$ while achieving extremely low call block, $B_{1}$, for class 1 . In some configurations it leads to a network utilization as high as Equalization. In all tests Equalization achieves a better link utilization but the price to pay is threshold management. The choice of acceptance threshold (which is beyond the scope of this paper) is far from 


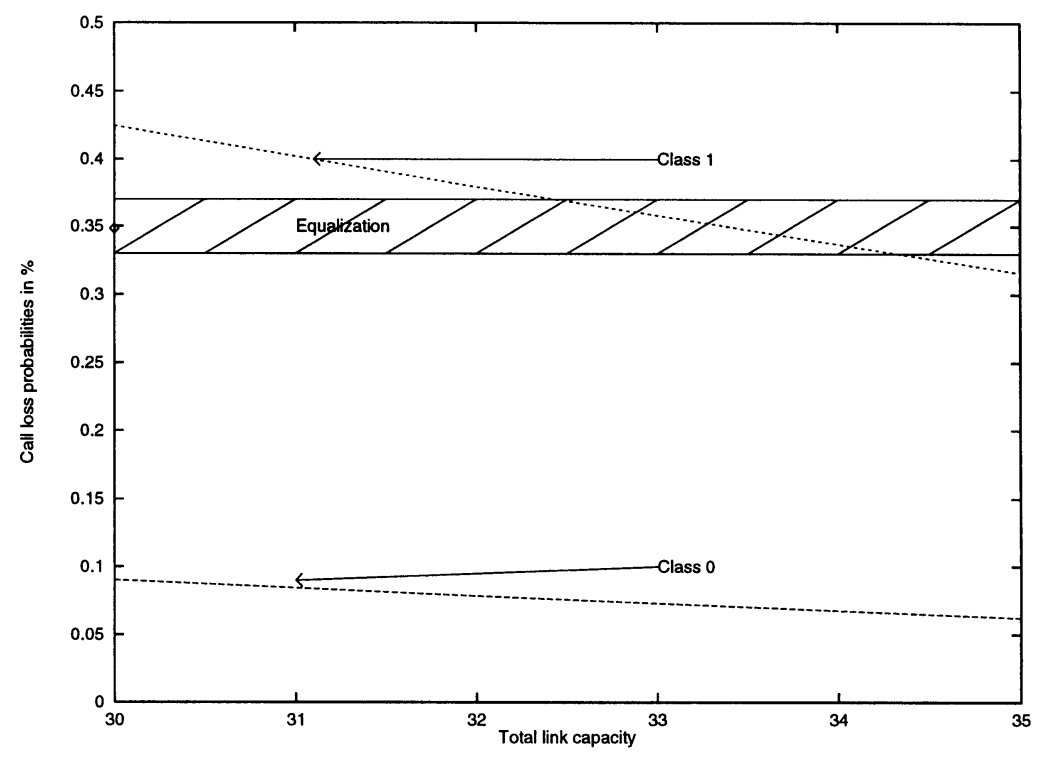

Figure 14: Total Capacity increase

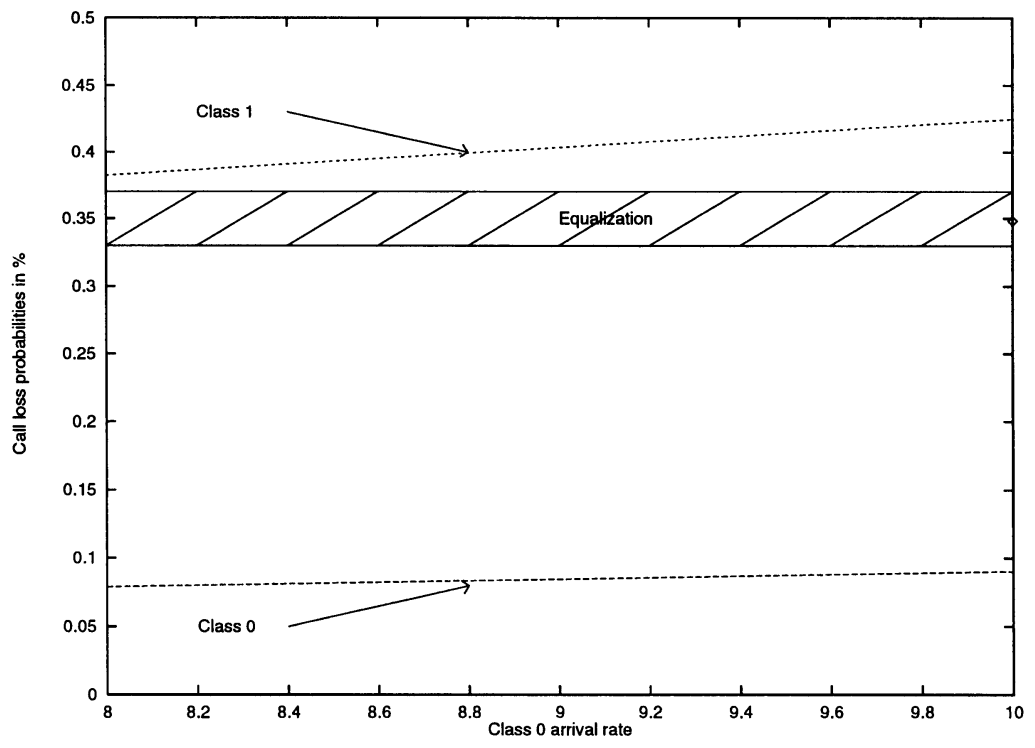

Figure 15: Arrival rate decrease 


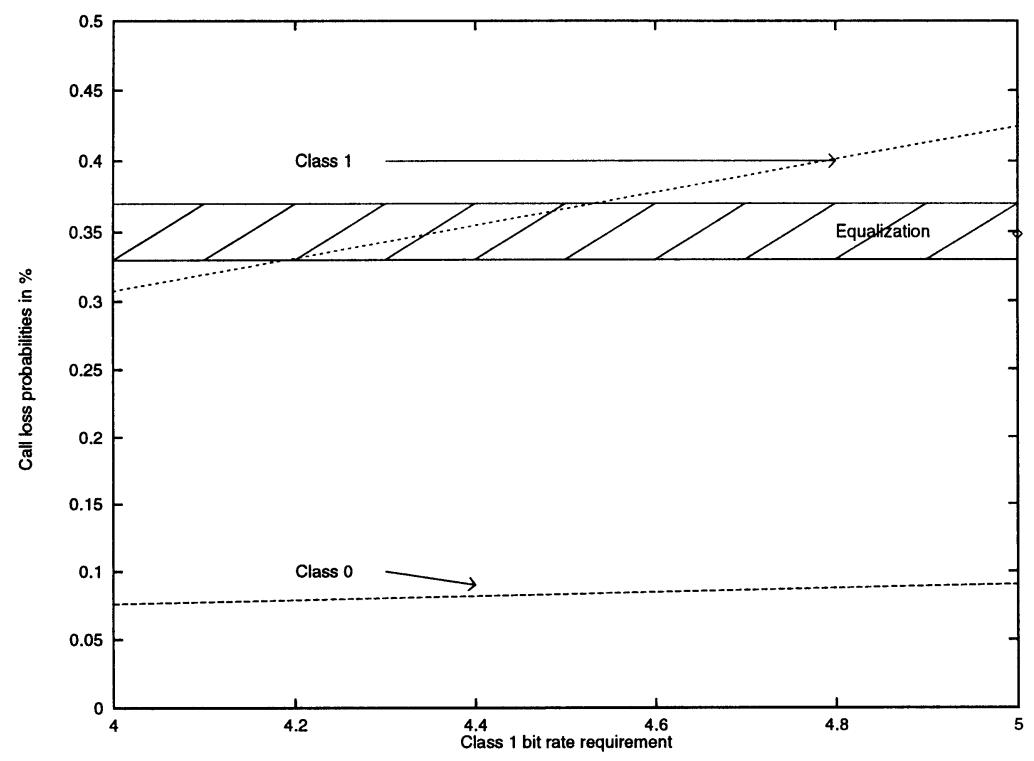

Figure 16: Bit rate requirements decrease

trivial in terms of implementation in a real size network. It amounts to know in advance all possible traffic classes, and to set up a GoS management policy among these classes. However, this additional complexity cost can be justified by the afforded capacity gains.

These results have been obtained with two different classes. Extensions to more than two classes are being pursued. In such configurations, more complex policies, in which the equalization principle applies to a subset of the population according to a class type selection or GoS level classification, must be derived.

The present study conducted in isolation considers only a single link. In order to use the equalization mechanism for CAC, equalization must be performed throughout the network on each stage of the path.

Future work will address all these issues.

\section{Acknowledgment.}

This work is supported by a FT-CNET grant (95IN01).

\section{References}

[RKK88] Ruth Kleinewillingnofer-Kopp, Eckart Wollner, Comparison of access control strategies for ISDN traffic on common trunk groups, ITC 12, Torino, June 1988. 
[EMi73] O. Enomoto, H. Miyamoto, An analysis of mixtures of multiple bandwidth traffic om time division switching networks, Proceedings of the 7th ITC, Stockolm 1973.

[DRo87] Z. Dziong, J.W. Roberts, Congestion probabilities in a circuitswitched integrated services network, Performance Evaluation 7, 1987.

[RTG94] Michael Ritter, Phuoc Tran-Gia, Multi-rate models for dimensioning and performance evaluation of ATM networks, COST 242, june 1994.

[ROB92] J. W. Roberts editor, COST 224, Performance evaluation and design of multi service network, Commission of the European communities, October 1992.

[RMV96] J. Roberts, U. Mocci, J. Virtamo Editors, Broadband Network Teletraffic, Performance evaluation and design ofn broadband multi services networks, Action COST 242, Springer- Verlag July 1996.

[Ros95] K.W. Ross, Multiservice Loss Models for Broadband Telecommunication Networks, Springer-Verlag, 1995.

[SkR93] S.Chung, K.W. Ross, Reduced load Approximations for Multirate Loss Networks, IEEE Transactions on Communications, Vol. 41, No.8, 1993. 